\title{
Model-based Monitoring of Septic Shock Treated with Large-pore Hemofiltration Therapy
}

\author{
James A. Revie*. David J. Stevenson*. J. Geoffrey Chase*. Bernard C. Lambermont**. Alexandre Ghuysen** \\ Philippe Kolh**. Geoffrey M. Shaw***. Thomas Desaive ${ }^{* *}$ \\ *Department of Mechanical Engineering, University of Canterbury, Chirstchurch, NZ \\ (e-mail: james.revie@pg.canterbury.ac.nz) \\ ** Hemodynamic Research Laboratory, University of Liege, Belgium \\ *** Department of Intensive Care, Christchurch Hospital, NZ
}

\begin{abstract}
The diagnostic ability of model-based monitoring systems offer significant clinical potential. This research tests, the clinical ability of a model-based system for tracking hemodynamic changes in a porcine study on septic shock and large pore hemofiltration (LPHF) therapy. Typically available or inferable intensive care unit (ICU) measurements are used to identify subject-specific models of the cardiovascular system (CVS). The approach accurately identified known trends of septic shock including a drop in systemic vascular resistance and right ventricular distension, and accurately predicted left and right ventricular volumes and pressures. The method also quantified each subject's individual response to the disease and corresponding LPHF therapy. These results indicate the clinical potential of using modelbased methods for tracking acute hemodynamic changes in cardiovascular compromised individuals.
\end{abstract}

Keywords: Parameter identification, physiological models, septic shock, hemodynamic monitoring.

\section{INTRODUCTION}

Septic shock is a highly prevalent disease in the intensive care unit (ICU) (Brun-Buisson et al., 1995). Cardiovascular management of septic shock can be difficult due to an array of complex circulatory interactions that occur within the patient and the limited number of available measurements to base treatment from. Model-based methods (Revie et al., 2011a, Revie et al., 2011b) can be used to aggregate common ICU measurements into an easy to understand physiological form, to paint a clearer picture of cardiovascular health and assist medical staff with therapy decisions.

This article tests the ability of a model-based method to track acute hemodynamic changes in an animal model of septic shock. Subject-specific models are retrospectively identified from measurements from a porcine study. In the experimental porcine study (Lambermont et al., 2006), an endotoxin infusion was used to induce septic shock and large pore hemofiltration (LPHF) therapy was applied. A range of hemodynamic measurements were recorded, and it was hypothesised that the filtration of small to medium sized water soluble cytokine molecules from the blood stream would reduce the systemic inflammatory effects of septic shock resulting in improved circulatory dynamics. However, the aim of this study is not to test the effectiveness of the LPHF, but to assess the ability of the model-based system to track the disease and therapy dependent hemodynamic changes.

The approach in this paper uses a parameter identification method to match a general model of cardiovascular system (CVS) to clinically available or inferable measurements obtained from the porcine study. Identified changes over time in subject-specific model parameters and model response are compared to clinically expected trends from the literature (Chan and Klinger, 2008, Dellinger, 2003) and independent measured data to test the accuracy and clinical applicability of the method. Hence, the overall goal of this work is to demonstrate the clinical relevance and prove the concept (and potential) of model-based clinical monitoring of: 1) CVS status; 2) acute dysfunction; and 3 ) the effect of treatment.

\section{METHODS}

\subsection{Porcine trials and measurements}

All procedures and protocols used were approved by the Ethics Committee of the Medical Faculty at the University of Liege, Belgium. Experiments were performed on 4 healthy pure pietrain pigs weighing between $20-30 \mathrm{~kg}$. The animals were premedicated, anesthetised, and ventilated as explained in (Lambermont et al., 2006). The animals received a 0.5$\mathrm{mg} / \mathrm{kg}$ endotoxin infusion over 30 minutes (from T0 to T30). From T60 onwards the pigs underwent a zero balance continuous veno-venous hemofiltration at a rate of 45 $\mathrm{ml} / \mathrm{kg} / \mathrm{h}$ with a $0.7 \mathrm{~m}^{2}$ large-pore (78 Angstrom) membrane with a cutoff of $80 \mathrm{kDa}$ and a BaxterBM 25-BM 14 hemofiltration device. Ultrafiltrate was replaced in the post dilution mode by a bicarbonate-buffered hemofiltration fluid at a temperature of $37^{\circ} \mathrm{C}$.

Micro-tipped catheters were used to record continuous hemodynamic measurements during the study. Every 30 minutes (T0 to T240) 6-12 heartbeats of the aortic and pulmonary artery pressure $\left(P_{a o}, P_{p a}\right)$, and the left and right ventricular pressure and volume waveforms were recorded at a $200 \mathrm{~Hz}$ sampling rate. $\left(P_{l v}, P_{r v}, V_{l v}, V_{r v}\right)$. In this study, 34 
sets of measurements from the 4 pigs were used. Two measurement sets for pig 1 at T30 and T60 were unusable. Further details on these trials can be found in (Lambermont et al., 2006).

\subsection{Cardiovascular system model}

A previously validated lumped parameter CVS model (Smith et al., 2004, Smith et al., 2005) describes the main global dynamics of the circulation, as shown in Fig. 1. The model acts a framework to which parameters can be personalised, to describe subject-specific hemodynamics. The lumped parameter structure is computationally relatively light, only requiring a small number of parameters, yet complex enough to describe all the clinically important hemodynamics.

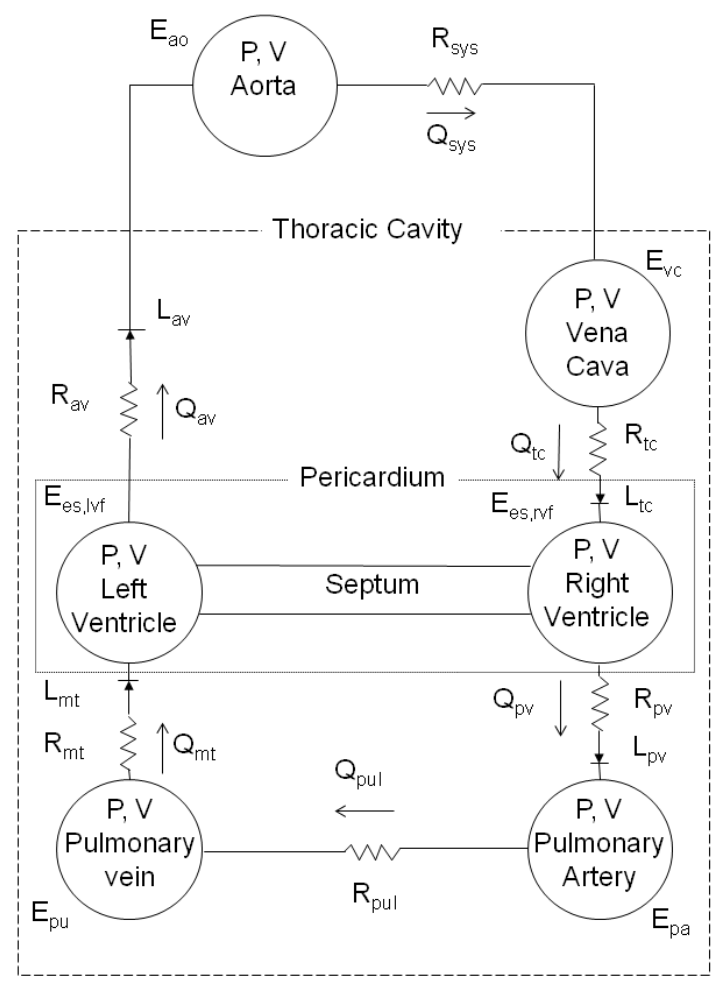

Fig. 1. Overview of the six-chamber CVS model.

The six-chamber model represents blood flow through the left and right ventricles, aortic and pulmonary arteries, and vena cava and pulmonary vein. Pressures and volumes $(\mathrm{P}, \mathrm{V})$ within these chambers and flows $(\mathrm{Q})$ between the chambers are defined using parameters of elastance $(E)$, resistance $(R)$, and inertia (L). Cardiac muscle activation is represented using time varying elastance, with the other non-cardiac chambers described using passive or constant elastance. Flow through the heart valves (mitral, aortic, tricuspid, and pulmonary artery) is controlled using one-way flow-gated valves which only allow forward flow. Furthermore, ventricular interaction is modelled through septal and pericardial dynamics. All these model features provide a means of accurately depicting the hemodynamics of the CVS.
A full list of the model equations and assumptions can be found in (Revie et al., 2011b).

\subsection{Parameter identification method}

In this study, an improved parameter identification method (Revie et al., 2011b) is used to replace the prior approach of (Starfinger et al., 2008). The previous method required knowledge of the left and right ventricular waveforms, which are expensive or highly invasive to measure continuously, and either the left and right ventricular pressures or population based assumptions for the valvular resistances. Hence, the method was difficult to apply in a clinical setting.

The approach used in this paper, requires only a small set of clinically available or inferable discrete measurements, as shown in Table 1, including the amplitude $\left(\mathrm{PP}_{\mathrm{ao}}, \mathrm{PP}_{\mathrm{pa}}\right)$, mean (MAP, MPAP), and maximum ascending gradient $\left(\mathrm{dP}_{\mathrm{ao}, \max }\right.$, $\left.\mathrm{dP}_{\mathrm{pa} \text {,max }}\right)$ of the aortic and pulmonary artery pressures, stroke volume (SV), global end diastolic volume (GEDV), and tricuspid and mitral valve closure times $\left(\mathrm{t}_{\mathrm{mt}}, \mathrm{t}_{\mathrm{tc}}\right)$. This set is much smaller, as it does not require full waveforms, and is far less intensive and invasive to obtain as the measurements are typically available already. Further, the new approach splits the identification process into parts, so only 2 or 3 parameters are identified at a time in smaller simplified models of the systemic and pulmonary circulations, increasing the convergence stability of the method, as explained in detail in (Revie et al., 2011a). Thus, this research improves the clinical applicability of the identification method without adding extra clinical effort or burden to the patient.

In this study, GEDV was assumed to be equal to the sum of the left and right ventricular end diastolic volumes. SV was taken as the average of the amplitudes of the left and right ventricular volume waveforms. The mitral and tricuspid valve closure times $\left(\mathrm{t}_{\mathrm{m}}, \mathrm{t}_{\mathrm{tc}}\right)$ were estimated from the derived subject-specific left and right ventricular driver functions, also known as time varying elastances. These driver functions were calculated using the method outlined in (Hann et al., 2011) from SV and features in the readily available aortic and pulmonary artery pressure waveforms.

During the identification process, all the resistances, elastances, and $P_{p u}$ and $P_{v c}$ in Table 1 are identified. The parameters of simplified systemic and pulmonary systems models are identified first. The left and right time varying elastance functions are used to simulate the simplified models, with initial guesses made for the parameter values. A proportional gain method is then used to identify the parameters of Table 1 by comparing the model outputs, which are most directly related to that parameter, to their corresponding clinical measurements, also shown in Table 1. Once the simplified models are identified, the parameters are joined together through identification of $E_{v c}$ and $E_{p u}$, to create a subject-specific CVS model. A more in depth description of the identification method can be found in (Revie et al., 2011b). 
Table 1: List of identified parameters and corresponding measurements used to identify them for the associated model. The parameter is shown on the same line as the measurement used to directly identify it.

\begin{tabular}{|c|c|c|c|c|}
\hline \multirow[t]{2}{*}{ Model } & \multicolumn{2}{|l|}{ Measurement s } & \multicolumn{2}{|l|}{ Identified Parameter } \\
\hline & Name & Symbol & Name & Symbol \\
\hline \multirow[t]{6}{*}{ Systemic } & Global end diastolic volume & GEDV & Left ventricular elastance & $E_{e s, l v f}$ \\
\hline & Stroke volume & SV & Mitral valve resistance & $R_{m t}$ \\
\hline & Mean aortic pressure & MAP & Systemic vascular resistance & $R_{s y s}$ \\
\hline & Aortic pulse pressure & $\mathrm{PP}_{\mathrm{ao}}$ & Aortic elastance & $E_{a o}$ \\
\hline & Maximum ascending aortic gradient & $\mathrm{dP}_{\mathrm{ao}, \max }$ & Aortic valve resistance & $R_{a v}$ \\
\hline & Mitral valve closure time & $\mathrm{t}_{\mathrm{mt}}$ & Pulmonary vein pressure & $P_{p u}$ \\
\hline \multirow[t]{6}{*}{ Pulmonary } & Global end diastolic volume & GEDV & Right ventricular elastance & $E_{e s, r v f}$ \\
\hline & Stroke volume & SV & Tricuspid valve resistance & $R_{t c}$ \\
\hline & Mean pulmonary artery pressure & MPAP & Pulmonary valve resistance & $R_{p u l}$ \\
\hline & Pulmonary artery pulse pressure & $\mathrm{PP}_{\mathrm{pa}}$ & Pulmonary artery elastance & $E_{p a}$ \\
\hline & $\begin{array}{l}\text { Maximum ascending pulmonary } \\
\text { artery pressure }\end{array}$ & $\mathrm{dP}_{\mathrm{pa} \text {,max }}$ & Pulmonary valve resistance & $R_{p v}$ \\
\hline & Tricuspid valve closure time & $t_{\text {tc }}$ & Vena cava pressure & $P_{v c}$ \\
\hline \multirow[t]{2}{*}{6 chamber } & Identified vena cava pressure & $\mathrm{P}_{\mathrm{vc}, \mathrm{sys}}$ & Vena cava elastance & $E_{v c}$ \\
\hline & Identified pulmonary vein pressure & $\mathrm{P}_{\mathrm{pu}, \mathrm{pul}}$ & Pulmonary vein elastance & $E_{p u}$ \\
\hline
\end{tabular}

\subsection{Statistical analysis}

Data is presented as mean $+/$ - standard error of the mean (SEM) unless stated otherwise. A paired-sample t-test was used to check temporal variance over T0 - T60 to analyse the effect of the endotoxin intervention. $\mathrm{P}<0.05$ was considered a statistically significant result. For validation, the relationship between the modelled and measured maximum left and right ventricular pressures and volumes were examined using correlation and linear regression analysis, including calculation of absolute percentage errors, and bias and precision analysis (Bland and Altman, 1986). These measurements were recorded experimentally, but importantly, were not used in the model identification process, and hence, provide independent validation of the method. A percentage error less than $20 \%$ was regarded as an acceptable result as measurement of physiological variables often lack precision, with typical errors of $+/-10-20 \%$ (Critchley and Critchley, 1999).

\section{RESULTS}

Subject-specific models were fitted to the 34 data sets recorded from the 4 pigs at $\mathrm{T} 0, \mathrm{~T} 30, \ldots, \mathrm{T} 210$ and $\mathrm{T} 240$. The models took an average 6 minutes to identify when run in Matlab on a single $2.8 \mathrm{GHz}$ core with $4 \mathrm{~GB}$ of memory. However, preliminary tests have indicated that models could be identified as fast as 3 seconds when compiled in C.

In this study, each animal acts as their own control with their baseline (T0) measurements reflecting the undiseased state of the pig. Statistically significant temporal changes $(p<0.05)$ were noticed in the measured systolic and diastolic aortic and pulmonary artery pressures, as well as in the right ventricular end diastolic volume over T0-T60. This result indicates that the endotoxin infusions have a significant pathological effect on the subject group. A summary of the main hemodynamic measurements is seen in Fig. 2.
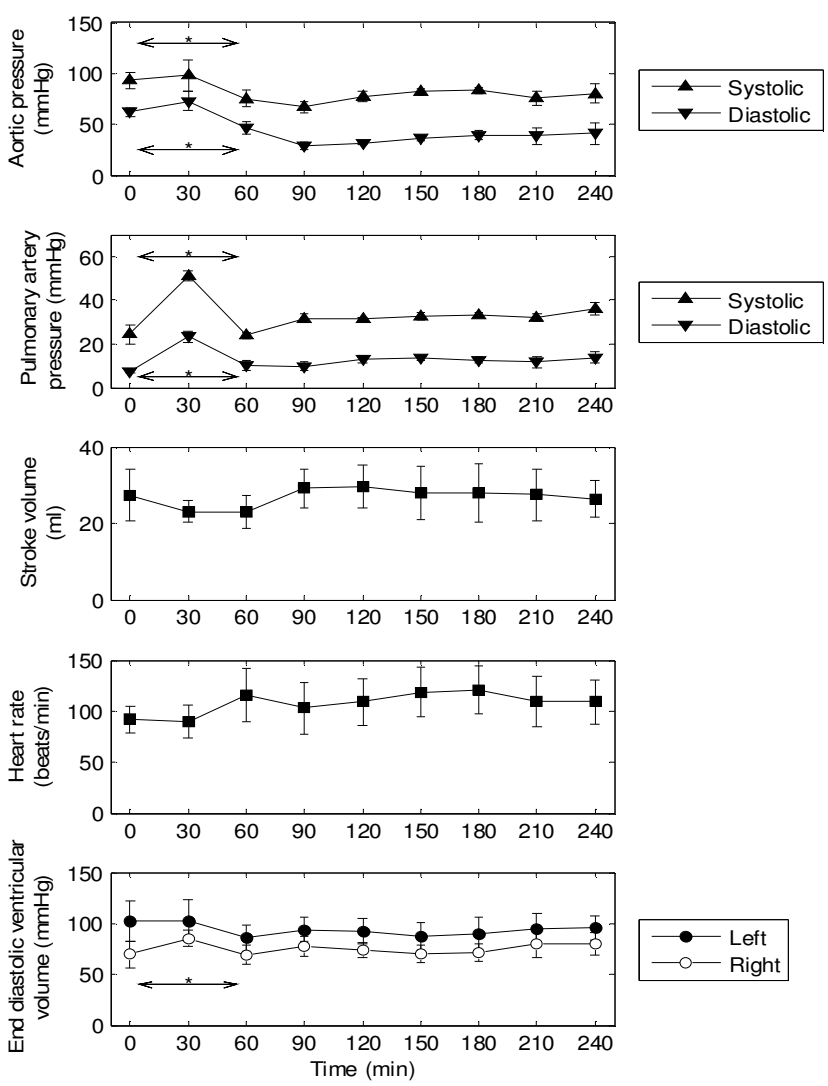

Fig. 2. Evolution of the averaged hemodynamic measurements recorded during the trials. * indicates $\mathrm{P}<0.05$ for expected temporal changes over T0-T60 due to the induction of septic shock. Data presented as mean +/- SEM.

\subsection{Validation of subject-specific models}

For all the data sets, the subject-specifc models matched the measuremeants used in the identification process including 
the mean aortic and pulmonary artery pressure, aortic and pulmonary artery pulse pressure and stroke volume, to percentage errors of less than $0.5 \%$., indicating successful convergence of the model identification method. Measurements that were not used for identifying the subjectspecific models, such as the left and right ventricular end diastolic volumes (LVEDV, RVEDV) and maximum left and right ventricular pressure $\left(P_{l v, \text { max }}, P_{r v, \text { max }}\right)$, were compared to their corresponding model outputs for validation. Bias, precision, and correlation metrics were analysed (Table 2). Small and opposite bias was noticed between the left and right ventricle measurements. The spread of the model outputs were less than $15 \mathrm{ml}$ and $11 \mathrm{mmHg}$ to 2 standard deviations (2SD). All four model outputs correlated well with the measured data, $\mathrm{R}^{2} \geq 0.78$. All percentage errors were within acceptable range $(<20 \%)$, except for the $95^{\text {th }}$ percentile error in the modelled maximum right ventricular pressure.

Table 2. Mean bias and precision metrics, median percentage errors with $5^{\text {th }}$ and $95^{\text {th }}$ percentile bounds, and correlation coefficients of the measurements used for validation of the model identification process.

\begin{tabular}{|l|l|l|l|}
\hline & Mean +/- 2SD & $\begin{array}{l}\text { \% Error } \mathbf{( 5}^{\text {th }}-\mathbf{9 5}^{\text {th }} \\
\text { percentile) }\end{array}$ & $\mathbf{R}^{\mathbf{2}}$ \\
\hline LVEDV & $-5.4+/-13.9 \mathrm{ml}$ & $7.5 \%(0.5 \%-13.8 \%)$ & 0.94 \\
\hline RVEDV & $4.9+/-14.1 \mathrm{ml}$ & $7.4 \%(0.5 \%-18.1 \%)$ & 0.91 \\
\hline $\mathbf{P}_{\mathrm{lv}, \max }$ & $-1.4+/-10.6 \mathrm{mmHg}$ & $4.5 \%(0.3 \%-15.2 \%)$ & 0.93 \\
\hline $\mathbf{P}_{\mathrm{rv}, \max }$ & $0.5+/-10.1 \mathrm{mmHg}$ & $6.2 \%(0.6 \%-26.6 \%)$ & 0.78 \\
\hline
\end{tabular}

\subsection{Septic shock trends}

The three main determinants of cardiovascular flow: afterload, preload, and contractility; are used to study the evolution of septic shock in this study. Afterload, the force required by the heart muscle to eject blood through the circulation, is investigated via analysis of the model parameters for systemic vascular resistance $\left(R_{\text {sys }}\right)$ and pulmonary vascular resistance $\left(R_{p u l}\right)$. Time varying changes in these parameters are shown in Fig. 3. Noticeably, there is a large drop in $R_{\text {sys }}$ and an increase in $R_{\text {pul }}$, symptomatic of septic shock.

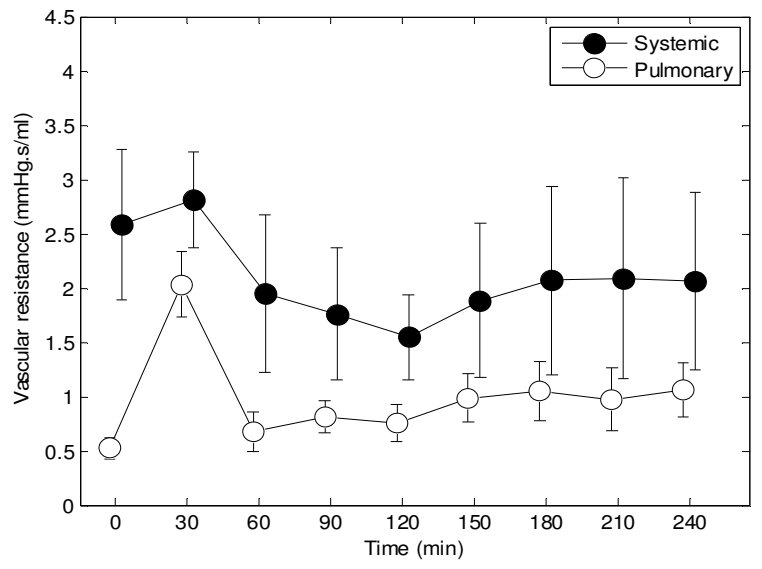

Fig. 3. Modelled left and right ventricular afterload. Data presented as mean $+/$ - SEM.
Preload, the force on the heart muscles during diastole, is represented in the model by the left and right ventricular end diastolic volume (LVEDV, RVEDV). LVEDV and RVEDV, averaged over all the pigs, is shown in Fig.4. RVEDV, increases during the study indicating the onset of right ventricle distension, a well known consequence of septic shock (Chan and Klinger, 2008).

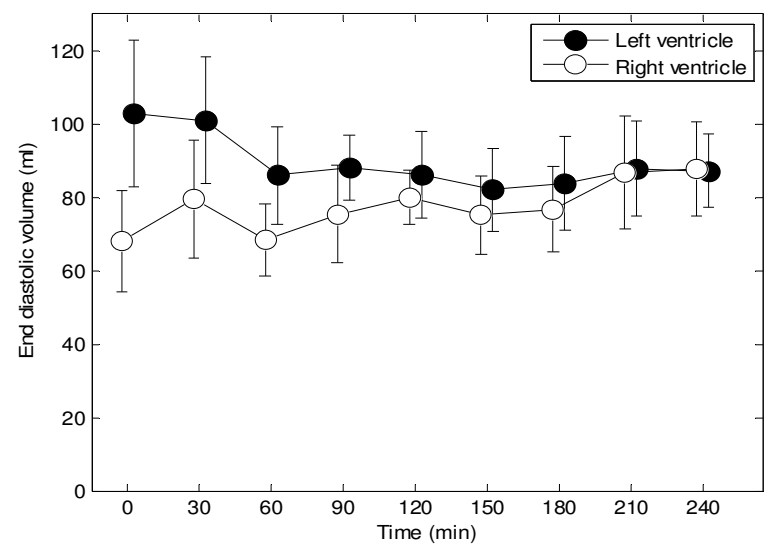

Fig. 4. Modelled left and right ventricular end diastolic volume (LVEDV, RVEDV). Data presented as mean +/SEM.

Lastly, contractility, the intrinsic ability of the heart muscle to contract independent of both preload and afterload, is defined in the model as the left and right ventricular end systolic elastance $\left(E_{e s, l v f}, E_{e s, r v f}\right)$. Changes in $E_{e s, l v f}$ and $E_{e s, r v f}$ over the duration of the study are shown in Fig. 5. A sharp spike in these parameters is seen initially after the endotoxin infusion, with $E_{e s, l v f}$ and $E_{e s, r v f}$ rising and then decreasing after T150.

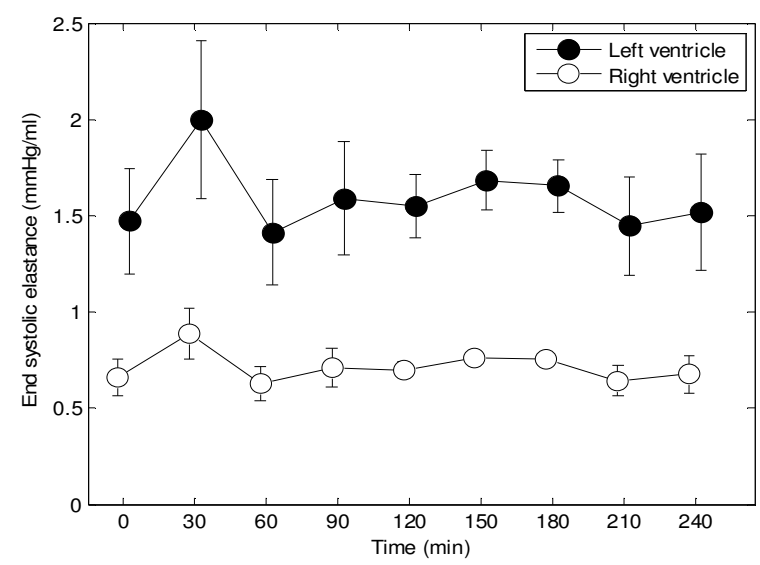

Fig. 5. Modelled left and right ventricular end systolic elastance $\left(E_{e s, l v f}, E_{e s, r v f}\right)$. Data presented as mean +/- SEM.

\subsection{Subject-specific response}

The averaged trends indicate that the model is able to track disease dependent changes resulting from septic shock across the 4 pigs. However, it is more important to understand the individual hemodynamics of each pig to assist with therapeutic decisions and tracking the progression of the disease. Hence, subject-specific $R_{s y s}$ is shown in Fig. 6 to 
compare with the averaged result (Fig. 3). On average, $R_{\text {sys }}$ drops by $20.3 \%$ across the four pigs over the duration of the trials. However, when analysing the individual response of each of the pigs, it is seen that the $R_{s y s}$ returns to baseline for Pig 2, and a substantial improvement is observed in Pig 1 after LPHF treatment (T60 onwards). This result shows how the model can be used to track the subject's individual response to the disease and LPHF treatment.

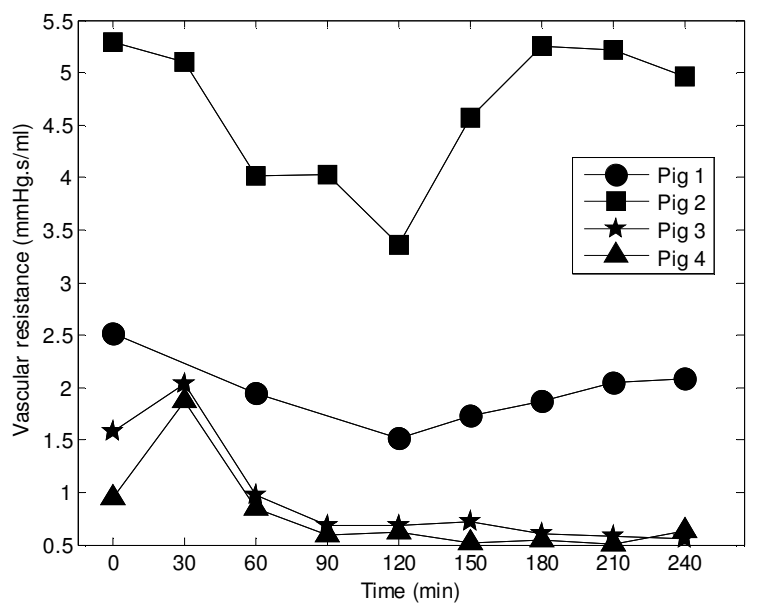

Fig. 6. Subject-specific, modelled systemic vascular resistance $\left(R_{\text {sys }}\right)$.

\section{DISCUSSION}

\subsection{Modelling errors}

For validation the model was compared to independent measurements (Lambermont et al., 2006) that were not used in the identification process. LVEDV and RVEDV were identified to a bias of less than $5.5 \mathrm{ml}$ and spread (2 SD) of less than $14.5 \mathrm{ml}$. The maximum left and right ventricular pressures were approximated to a bias and spread (2 SD) of less than $1.5 \mathrm{mmHg}$ and $11 \mathrm{mmHg}$, respectively. The time varying trends in these four measurements were matched to a high degree of accuracy, with $\mathrm{R}^{2}>0.90$, except for the maximum right ventricular pressure with $\mathrm{R}^{2}=0.78$, which is still a good result. Importantly, all ventricular volumes were identified to an error of less than $20 \%$ (except for the $95^{\text {th }}$ percentile error in the maximum right ventricular pressure, which is acceptable for this type of physiological monitoring (Critchley and Critchley, 1999).

A primary cause of the errors in Table 2, especially the fact the errors are of similar magnitude but opposite polarity, is due to the difficulties in measuring the right ventricle volume with a conductance catheter. The complex shape of the right ventricle can lead to underestimation of the volume waveform. When comparing left and right ventricular SV it was noticed that in many data sets the left SV was consistently greater than two times the right SV, which cannot physiologically be maintained for more than a few heartbeats. The SV of both ventricles in the CVS model is assumed to be equal so an average of the measured left and right ventricular SVs was used in the identification process. This fact caused the left ventricular volume, and consequentially, the left ventricular pressure to be overestimated, with the opposite occurring in the right ventricle. However, more importantly, these errors were systematic so the trends associated with these measurements are still clearly identified and accurate.

\subsection{Detecting septic shock}

The subject-specific models of the CVS captured the main hemodynamic trends of septic shock. A drop in systemic vascular resistance, corresponding to a loss of vascular tone in the arterial system (Chan and Klinger, 2008, Dellinger, 2003), and an increase in pulmonary vascular resistance were noticed, a known result of the disease (Chan and Klinger, 2008, Dellinger, 2003). Initially, for Pigs 3 and 4 a decrease in $R_{s y s}$ in Fig. 6 leads to an increase in cardiac output, reflective of warm septic shock. Later in the trials, the decreasing ratio of the left to right ventricular afterload causes dilation of the right ventricle and a decrease in left ventricular preload (LVEDV). These two factors would have resulted in a flattening of the intra-ventricular septum and a decrease in the left ventricular function (Chan and Klinger, 2008). This point is reinforced through analysis of left ventricular contractility, $E_{e s, l v f}$, which increases during the middle stage, but starts to drop in the later stage of the trials. Hence, the model accurately captures the expected trends of septic shock in the pigs.

\subsection{Large pore hemofiltration therapy}

Large pore hemofiltration appears to have a positive effect on pigs 1 and 2, where $R_{s y s}$ begins to increase one hour after the treatment has started (T120). Hence, mean aortic pressure increases in these two pigs independent of changes in cardiac output. there are negligible positive changes in pigs 3 and 4, where decreases in mean aortic pressure are not reversed by increases in flow. These results tentatively suggest that LPHF is responsible for increasing systemic resistance in two of the pigs, but appears to have little effect on the other two. Larger trials are required before a more substantiated conclusion on this therapy can be made, which was not the goal of this research. However, it is clear the model has accurately segregated those subjects who did (and did not) respond positively to the endotoxin insult, due either to internal cardiovascular reflexes or because of the effects of this therapy.

\subsection{Limitations}

The main limitation of the model identification process is its reliance on the knowing pulmonary artery pressure. In recent times the use of the pulmonary artery catheter (PAC) has decreased (Binanay et al., 2005, Shah et al., 2005), as its use has been associated with little benefit, and even detrimental at times, to patient outcomes (Shah et al., 2005). However, accurate diagnosis and monitoring of critical hemodynamics, as demonstrated here, could reverse that trend turning potential risk into benefit. Hence, the ability of this CVS model and method to turn a set of clinical data into a clearer, 
accurate physiological picture could justify the use of these devices by adding currently unrealised value to the data they deliver.

Other limitations revolve around the relatively simple lumped parameter nature of the CVS model. Due to this simplicity the model is unable to capture CVS micro dynamics of the CVS. For example, the aortic notch in the aortic pressure waveform cannot be modelled. However, the model describes all the major CVS dynamics, such as changes in ventricular preload, afterload, and inotropy, which can be altered and controlled with current therapies. Further, due to the lumped parameter structure, only a small number of parameters are required to define the CVS model, resulting in the model being identifiable from the minimal measurement set available in the ICU.

\section{CONCLUSION}

The subject-specific models were capable of accurately identifying all the key determinants of cardiovascular health, including afterload, preload, and contractility. The method tracked known disease and treatment dependent changes in the pigs resulting from the endotoxin infusion and later LPHF treatment. These results show that this model-based monitoring technique can be used to accurately identify and discriminate clinically useful cardiac and circulatory information in acute CVS dysfunction. Such a method could be implemented in intensive care at little extra cost as it only available or easily inferable in the ICU, and would be of great benefit as it can provide accurate real-time feedback to medical staff on a patient's cardiovascular health.

\section{REFERENCES}

Binanay, C., Califf, R. M., Hasselblad, V., O'Connor, C. M., Shah, M. R., Sopko, G., Stevenson, L. W., Francis, G. S., Leier, C. V. \& Miller, L. W. (2005) Evaluation study of congestive heart failure and pulmonary artery catheterization effectiveness: the ESCAPE trial. JAMA : the journal of the American Medical Association, 294, 1625-33.

Bland, J. M. \& Altman, D. G. (1986) Statistical methods for assessing agreement between two methods of clinical measurement. Lancet, 1, 307-10.

Brun-Buisson, C., Doyon, F., Carlet, J., Dellamonica, P., Gouin, F., Lepoutre, A., Mercier, J. C., Offenstadt, G. \& Regnier, B. (1995) Incidence, risk factors, and outcome of severe sepsis and septic shock in adults. A multicenter prospective study in intensive care units. French ICU Group for Severe Sepsis. Jama, 274, 968-74.

Chan, C. M. \& Klinger, J. R. (2008) The right ventricle in sepsis. Clin Chest Med, 29, 661-76, ix.

Critchley, L. A. \& Critchley, J. A. (1999) A meta-analysis of studies using bias and precision statistics to compare cardiac output measurement techniques. Journal of clinical monitoring and computing, 15, 85-91.
Dellinger, R. P. (2003) Cardiovascular management of septic shock. Critical care medicine, 31, 946-55.

Hann, C. E., Revie, J., Stevenson, D., Heldmann, S., Desaive, T., Froissart, C. B., Lambermont, B., Ghuysen, A., Kolh, P., Shaw, G. M. \& Chase, J. G. (2011) Patient specific identification of the cardiac driver function in a cardiovascular system model. Computer methods and programs in biomedicine, 101, 201-7.

Lambermont, B., Delanaye, P., Dogne, J. M., Ghuysen, A., Janssen, N., Dubois, B., Desaive, T., Kolh, P., D'Orio, V. \& Krzesinski, J. M. (2006) Large-pore membrane hemofiltration increases cytokine clearance and improves right ventricular-vascular coupling during endotoxic shock in pigs. Artificial organs, 30, 560-564.

Revie, J. A., Stevenson, D. J., Chase, J. G., Hann, C. E., Lambermont, B. C., Ghuysen, A., Kolh, P., Morimont, P., Shaw, G. M. \& Desaive, T. (2011a) Clinical detection and monitoring of acute pulmonary embolism: proof of concept of a computer-based method. Ann Intensive Care, 1, 33.

Revie, J. A., Stevenson, D. J., Chase, J. G., Hann, C. E., Lambermont, B. C., Ghuysen, A., Kolh, P., Shaw, G. M., Heldmann, S. \& Desaive, T. (2011b) Validation of subject-specific cardiovascular system models from porcine measurements. Computer methods and programs in biomedicine, in press.

Shah, M. R., Hasselblad, V., Stevenson, L. W., Binanay, C., O'Connor, C. M., Sopko, G. \& Califf, R. M. (2005) Impact of the pulmonary artery catheter in critically ill patients: meta-analysis of randomized clinical trials. JAMA : the journal of the American Medical Association, 294, 1664-70.

Smith, B. W., Chase, J. G., Nokes, R. I., Shaw, G. M. \& Wake, G. (2004) Minimal haemodynamic system model including ventricular interaction and valve dynamics. Medical engineering \& physics, 26, 1319.

Smith, B. W., Chase, J. G., Shaw, G. M. \& Nokes, R. I. (2005) Experimentally verified minimal cardiovascular system model for rapid diagnostic assistance. Control Engineering Practice, 13, 1183 1193.

Starfinger, C., Chase, J. G., Hann, C. E., Shaw, G. M., Lambermont, B., Ghuysen, A., Kolh, P., Dauby, P. C. \& Desaive, T. (2008) Model-based identification and diagnosis of a porcine model of induced endotoxic shock with hemofiltration. Mathematical biosciences, 216, 132-9. 